\title{
Preparation and properties of injectable hemostatic and wound healing promoting hydrogel
}

\author{
Keru Tang ${ }^{1}$ \\ ${ }^{1}$ Beijing National Day School, No 66, Yuquan Road, Haidian District, Beijing, China
}

\begin{abstract}
In daily life, people are susceptible to injuries from the outside world. Bleeding wounds and longterm non-healing bring a lot of trouble to the lives of the injured. As a result, it developed a class of wound healing materials based on gelatin combined with calcium ions. By exploring the different behaviors of gelatin aqueous solution at different temperatures and concentrations to form gels, we determined the concentration of the prepared gelatin. Next, calcium-containing substances were added to the gelatin. Through discussing the way and concentration of two different forms of calcium source eggshell and calcium gluconate added to gelatin, the optimal ratio of calcium source to gelatin was determined. Finally, an injectable gelatin hydrogel hemostatic material which is convenient for coating was prepared.
\end{abstract}

\section{Introduction}

The traditional hemostatic materials currently applied in clinical practice have certain limitations, such as: 1) They cannot actively accelerate healing, and at the same time, they tend to adhere to the blood tissue of the wound, which causes the wounded to be very painful when removed. For instance, topical gauze is often mixed with tissue fluid and cannot be removed from the wound after it dries; 2) Although the wound can be wrapped with band-aids to prevent the wound from contacting with external substances, the air-permeability of the band-aid is not good, and once the wound is dipped in water, it will fall off easily, causing wound infection.

Currently, the auxiliary materials that boost wound healing reported in the literature mainly contain inorganic materials and macromolecule materials, as well as some multifunctional new composite materials. Inorganic materials mainly include traditional calcium carbonate, etc., as well as metal nanoparticles such as silver, gold, copper, and metal oxide particles in recent years [1]. These materials have excellent antibacterial activity, which can accelerate wound healing and suppress bacterial growth during wound healing. Macromolecular hemostatic materials contain natural macromolecules such as chitosan and collagen, as well as synthetic macromolecule materials [2-3]. For instance, chitosan has good biocompatibility, hemostatic and antibacterial effects. However, single-component hemostatic materials still have certain limitations. Hence, if different kinds of materials are combined into a multifunctional new composite material, various properties of the material can be significantly improved.

\subsection{Materials for promoting wound healing}

In the early days, people have been looking for some natural materials from nature to help wounds heal. In the field of traditional medicine in our country, many natural animal and plant medicines have been prepared into various hemostatic and wound healing medicines. For instance, Panax notoginseng can help stimulate blood clotting factors, The traditional Chinese medicine rhubarb can boost the concentration of platelets and decrease the permeability of blood vessels [7]. Eaxcept herbs, some minerals also have a good hemostatic effect, such as stamen, red stone fat, etc. [7].

In modern medicine, various hemostatic materials have been developed. For example, in the 1980s, the US military utilized zeolite powder for auxiliary hemostasis after soldiers were injured on the battlefield. However, the application of such materials has been limited because zeolite emits a lot of heat during hemostasis, leading to burns. Since then, people have developed a series of biomedical macromolecule materials to help stop bleeding [8-9]. Besides, some inorganic compounds also have a good hemostatic effect, such as zinc oxide powder, which has a wide range of clinical use in the field of burns and scalds [10]. However, some of these hemostatic materials are inconvenient to use because many of them are in powder form and are not easy to fix on the wound. At the same time, if a wound occurs, the coating method is not very convenient.

Currently, the newly developed inorganic materials for accelerating wound healing include nanomaterials developed one the basis of metals such as silver, gold, copper, cerium. in addition to nanoscale bioactive glass, $\mathrm{ZnO}$ nanomaterials, carbon nanomaterials, titanium dioxide nanomaterials, etc. [11-18]. These nanomaterials

*Corresponding author: dingli@niesl.com.cn 
can produce reactive oxygen species, which can then be compounded with other materials to prevent microorganisms from attaching to DNA and RNA to multiply. They have good hemostasis and bactericidal effects.

For example, silicon dioxide (SiO2) coated goldsilver nanomaterials can release $\mathrm{Ag}+$ slowly through photothermal properties under the excitation of nearinfrared lasers. The porous and thin-walled feature allows Ag+ to be released. The excitation of the nearinfrared laser enables the material to produce photothermal effect, which has an antibacterial effect on Staphylococcus aureus and Escherichia coli, thereby facilitating wound healing [15]. Moreover, calcium ion is also a very good substance for wound healing, because it is an activator of many prothrombin and can also be directly applied as a coagulation complex factor [19].

\subsection{Macromolecular materials for promoting wound healing}

The most commonly utilized materials for promoting wound healing are macromolecule materials, including natural macromolecule materials and synthetic macromolecules. Currently, the main natural macromolecules that have been developed to accelerate wound healing contain polysaccharide structures such as chitosan, cellulose, and hyaluronic acid, as well as protein-like collagen, silk protein, honey and other macromolecules. Due to its good biocompatibility, biodegradability, and cell/tissue adhesion, the role of macromolecule materials in boosting cell migration, proliferation, and differentiation in the process of wound healing has been extensively investigated.

Gel is a three-dimensional network structure formed by polymer in solvent. The appearance of the whole material is uniform and has a certain shape of elastic solid [20]. In the gel system, polymers are connected to each other through cross-linking points to form a spatial network structure, and small solvent molecules can diffuse into the polymer network. Common gels in daily life contain jelly, pigskin jelly, and some common dosage forms for cosmetics.

Injectable gels are a type of hydrogel that can be injected with a syringe. Compared with traditional injections, injectable gel is more convenient to utilize. It not only can be employed as a physical network structure of a three-dimensional matrix, but also can achieve controlled release of drugs. Injectable gel can be easily coated on solid surfaces by extrusion and has a good application prospect in hemostatic materials [21].

Gelatin is a type of macromolecular protein extracted from animal leather with similar properties to macromolecular proteins, and has a wide range of applications in food, medicine and other fields [23]. When dissolved in water at a higher temperature, gelatin is in a sol state, that is, a liquid with a larger viscosity. After the high-temperature gelatin sol is cooled, a gel with higher elasticity is formed, which has good elasticity and water content. However, when the low- temperature gelatin sol is heated again, it can be dissolved again to become a sol.

As a result, It can develop a wound-healing promoting material that is convenient to apply based on gelatin materials. It can be extruded and applied to the wound like toothpaste, and then spread evenly to form a film. This will not only affect the freedom of movement of the injured, but can also play a part in stopping bleeding and promoting wound healing. After the wound is healed, the material can be washed away with water. As a common substance in life, calcium ions can help to activate prothrombin. Hence, calcium ions were mixed with gelatin to prepare a new type of hemostatic material in this study.

\section{Experimental materials}

Vinegar with acetic acid content about $6 \%$, sodium carbonate, eggshell, gelatin powder, purified water, calcium gluconate oral liquid.

\subsection{The configuration and the gelation process of different concentrations of gel solution}

Pure water was added to $12 \mathrm{~g}$ of gelatin powder until the total mass of the mixture reached $75 \mathrm{~g}$. In a water bath at $80{ }^{\circ} \mathrm{C}$, the gelatin powder was completely dissolved in water to prepare a $16 \%$ solution. Then, the solution was diluted with water to a concentration of $8 \%, 4 \%, 2 \%$, and $1 \%$, and a total of $16 \%, 8 \%, 4 \%, 2 \%, 1 \%$, and $0.05 \%$ gelatin solutions were acquired. Next, let it stand for $10 \mathrm{~h}$ at a temperature of $4{ }^{\circ} \mathrm{C}$ to gelatinize the gelatin solution.

The initial state of gelatin was powder. Different quality of gelatin was added to water at $80{ }^{\circ} \mathrm{C}$ until it was dissolved. The resulting gelatin solution was then allowed to stand overnight at $4{ }^{\circ} \mathrm{C}$. Then, the $16 \%, 8 \%$, $4 \%, 2 \%$, and $1 \%$ gelatin solutions became gels and would not flow down when placed in a glass bottle. However, the $0.05 \%$ gelatin solution could still flow down even after being left overnight at low temperature and inverted, indicating that no gel was formed.

The solution prepared at $80{ }^{\circ} \mathrm{C}$ was sucked into the syringe and placed at $4{ }^{\circ} \mathrm{C}$ for $10 \mathrm{~h}$. Push the syringe and feel for resistance. The $1 \%$ and $2 \%$ solutions are the same as liquid water and have no adhesion during injection. It pushed the syringe and felt for resistance. The $1 \%$ and $2 \%$ solutions were the same as liquid water and had no adhesion during injection. $4 \%$ and $8 \%$ gels were more suitable as the ratio of injectable gels because they were more adhesive. Besides, the syringe filled with $4 \%$ gel had resistance but was not difficult to push. The syringe filled with $8 \%$ gel had some resistance that interfered with injection. However, $16 \%$ of the gel was too hard to be ejected from the syringe. As a result, $4 \%$ gel was more suitable for injectable gel. 


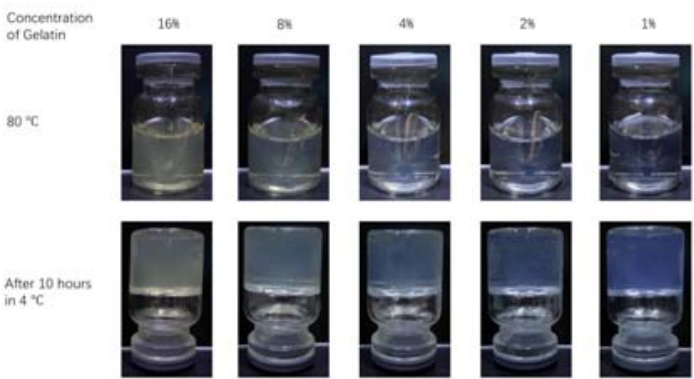

Figure 1 Different concentrations before and after solidification

\subsection{Introduction of calcium source}

The most familiar source of calcium in daily life is eggshells, because eggshells are rich in calcium carbonate. According to the reaction equation of acetic acid and calcium carbonate, it can be known that calcium ions can be dissolved in acetic acid. The assay we initially designed aimed to acquire calcium ions from egg shells and the specific reaction equation is as follows: $2 \mathrm{CH} 3 \mathrm{COOH}(\mathrm{aq})+\mathrm{CaCO} 3(\mathrm{~s})=\mathrm{Ca} 2+(\mathrm{aq})+$ $\mathrm{H} 2 \mathrm{O}+2 \mathrm{CH} 3 \mathrm{COO}-+\mathrm{CO} 2$

As expressed in the schematic diagram, it designed two methods to introduce calcium ions. The first method: by dissolving solid calcium carbonate in an acidic solution, free calcium ions were obtained. Then, it was mixed with the gelatin solution and the sodium carbonate solution was added to precipitate calcium ions, thereby obtaining a sol-like mixed sol of calcium carbonate and gelatin. The second method: firstly, a sol-like calcium carbonate was prepared. Then it was mixed with a gelatin solution to gain a gelatin sol containing calcium carbonate.

In terms of these two assays, many small bubbles were generated when the eggshell was placed in the vinegar. After a long enough time, the eggshell was still not dissolved, but its hardness was decreased. As proved by the experimental results, the method of utilizing white vinegar to dissolve egg shells cannot gain free calcium ions from egg shells.

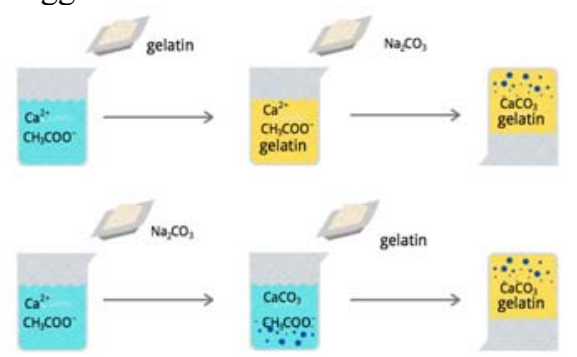

Figure 2 Schematic diagram of introducing calcium ions from eggshells into the gel system

\subsection{Calcium ions introduction with calcium gluconate oral liquid}

It was indicated that using egg shells was not effective in introducing calcium ions into the gel system. In an effort to successfully introduce calcium ions, calcium gluconate oral liquid was employed instead. The calcium gluconate oral solution contains $99 \mathrm{mg} / \mathrm{mL}$ calcium ions. This solution was utilized to replace the $4 \%$ gelatin solution prepared in all the water in assay 1, making it gel. The gelation performance was tested. The gel obtained in the assay had no evident difference in hardness and injectability from the samples prepared with water.

\subsection{Film formation assay}

Regarding gel-coated wounds, the film properties of the gel are crucial. In an interest of testing the film-forming ability of solutions of various concentrations, the solution prepared in assay 3 was applied for film formation experiments.

First of all, a plastic board was placed horizontally on the table, and five $3 * 5 \mathrm{~cm}$ squares were coated on the plastic board. At room temperature, a syringe and a cotton swab were utilized to smear $1 \mathrm{~mL}$ of a gel solution containing calcium gluconate with different concentrations on the plastic plate. The plastic plate was placed perpendicular to the ground, and then the gelation state of gel solutions of different concentrations was observed.

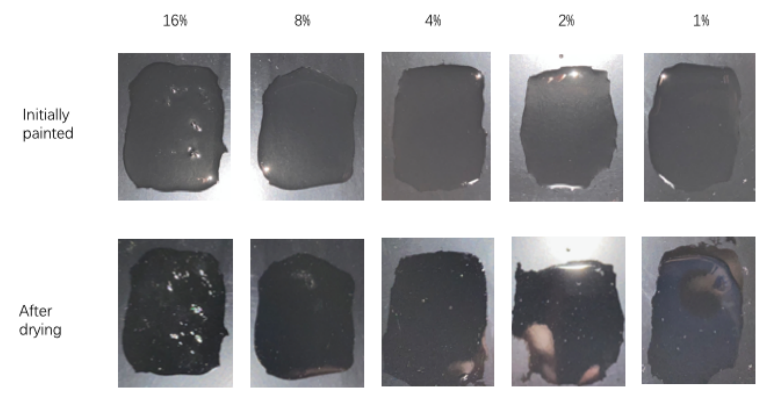

Figure 3 Different concentrations of gel solutions

After being applied to the leather, the $16 \%$ and $8 \%$ gel solutions immediately formed a gel, however, the $4 \%$, $2 \%$, and $1 \%$ gel solutions remained in the sol state and might flow down from the leather. Besides, $4 \%, 2 \%$ and $1 \%$ gel solution formed a gel after $30 \mathrm{~min}, 1 \mathrm{~h}$, and $2 \mathrm{~h}$, respectively.

High-concentration gel solutions of $16 \%$ and $8 \%$ showed better gelation ability even at room temperature, due to the fact that more gel macromolecules could easily form a network. While the $4 \%, 2 \%$, and $1 \%$ gel solutions contain more water molecules, which reduce the ability to gel.

\subsection{Hemostasis assay}

Since it accidentally scratched my arm, I took the opportunity to try to stop the bleeding with the gel I made. When applied, the gel formed a film on the skin that stopped bleeding and could be peeled off when it dried. 


\section{Conclusion}

It configured gelatin solutions of different concentrations, and studied the gel properties of gelatin solutions at different concentrations and different temperatures and the transition between gel and sol at the same time. It was revealed that $4 \%$ gelatin solutions had moderate gel properties. Then, put the egg shell in white vinegar, aiming to dissolve the egg shell to acquire the calcium source. However, due to the low concentration of vinegar, the egg shells were not dissolved. As a result, the method of introducing calcium source failed. In an effort to introduce calcium ions into the gel, I applied calcium gluconate solution again. By dissolving the gelatin with calcium gluconate solution, the gelatin containing calcium ions was gained. The introduction of calcium ions could enhance the hemostatic ability of the gel. Next, I explored the film-forming properties of gelatin gel. The gelatin gel was extruded through a syringe and coated on the plastic to obtain gel films of different thickness and hardness. Finally, it was indicated that $4 \%$ gelatin gel had good properties. At the same time, the hardness of the film after film formation was moderate and easy to remove. Hence, the $4 \%$ concentration of calcium ion-containing gelatin gel is expected to be a kind of good hemostatic material.

\section{References}

1. Liu Liu, Qingzhu Zhou, Zhuo Gong, Boyan Liu, Bin Yang, Xian Zhao. Characteristics and manufacturing technology of collagen/inorganic materials to construct tissue engineering bone $[\mathrm{J}]$. China Tissue Engineering Research, 2021,25 (04): 607-613.

2. Chen Zhao, Haoqun Hong, Jia Liu, Junjie Cai, Liangang Xiao, Wanyi Tan, Yidong Liu. Teaching exploration of research-oriented polymer materials and engineering specialty experiments under the background of new engineering [J]. Guangdong Chemical Industry, 2020,47(16): 239+254.

3. Jing Zhang,Zhi Li,Xiao-Lin Qi,De-Yi Wang. Recent Progress on Metal-Organic Framework and Its Derivatives as Novel Fire Retardants to Polymeric Materials[J]. Nano-Micro Letters,2020,12(1).

4. Jun Deng. Research on the use of nanomaterials to kill drug-resistant bacteria and promote wound healing $[\mathrm{C}]$. Antibacterial Industry Branch of National Health Industry Enterprise Management Association, School of Life Science and Technology, Beijing University of Chemical Technology, Beijing Laboratory of Biomedical Materials. 2018 (3rd) Antimicrobial Science and Technology Forum Paper Abstract Collection. Antibacterial Industry Branch of National Health Industry Enterprise Management Association, School of Life Science and Technology, Beijing University of Chemical Technology, Beijing Laboratory of Biomedical Materials: Antimicrobial Industry Branch of National Health Industry Enterprise Management Association, 2018: 289.
5. Weilu Cheng. Preparation and performance study of natural polymer-based multifunctional hemostatic composite dressing [D]. Harbin Institute of Technology, 2016.

6. Guangqian Lan. Development and performance study of chitosan/gelatin composite hemostatic material [D]. Southwest University, 2016.

7. Jingting Gao. Preparation and biological activity of chitosan-zinc oxide composite antibacterial dressing [D]. Wuhan University of Technology, 2015.

8. Yanhong Zhou, Tong Hao, Junjie Li, Lanlan Feng, Wenhui Zhang. Development and biological effect evaluation of a new nano-silver-based anti-infective dressing [J]. Military Medicine, 2016, 40 (06): 497501.

9. Yang Li. Preparation and performance study of regenerated silk fibroin silver-loaded wound dressing [D]. Chongqing Medical University, 2020.

10. Ulanhas. Development and preliminary experimental study of hollow activated carbon fibernano-silver composite dressing [D]. Second Military Medical University, 2009.

11. Jing Cui. Research on electrospun PVA-SbQ/zein drug-loaded nanofiber wound healing material [D]. Jiangnan University, 2015.

12. Yuyu Qiu. Structure construction of multi-layer composite wound dressing and its effect on wound healing mechanism [D]. Jiangnan University, 2019.

13. Xingze Li. Preparation of Sesamol-CA/Zein Composite Nanofiber Membrane and Evaluation of Wound Healing Function [D]. Northwest Sci-Tech University of Agriculture and Forestry, 2019.

14. Bettencourt Ana,Gonçalves Lídia M,Gramacho Ana C,Vieira Adriana,Rolo Dora,Martins Carla,Assunção Ricardo,Alvito Paula,Silva Maria João,Louro Henriqueta. Analysis of the Characteristics and Cytotoxicity of Titanium Dioxide Nanomaterials Following Simulated In Vitro Digestion.[J]. Nanomaterials (Basel, Switzerland),2020,10(8).

15. Weizong Weng. Study on the treatment of infectious bone defects and wound scars after open fractures with carbon nanomaterials [D]. Second Military Medical University, 2017.

16. MENG Hui-ping; LI Dong-li; Yang Yan-ze, Calcium and Human Health, Studies of Trace Elements and Health, Vol. 27, No.5, 2010.9, 65-67

17. Zhenkun Zhang, Zhe Li, Ya Li, Yingying Wang, Yaping Wang, Xinkui Zhou, Shanshan Ma, Fangxia Guan. Application of alginate-based hydrogel/dressing in wound healing: continuous, dynamic and sequential release $[\mathrm{J}]$. China Tissue Engineering Research, 2021, 25 (04): 638-643.

18. Hui Zhang. Preparation of zincbased/polyquaternium-based antibacterial hydrogel and its use as a wound dressing [D]. Qingdao University, 2019.

19. https://m.sohu.com/a/191206111_609505/ 
20. Marina Czerner,Mariano Prudente,Josefa Fabiana Martucci,Federico Rueda,Laura Alejandra Fasce. Mechanical behavior of cold-water fish gelatin gels crosslinked with 1,4-butanediol diglycidyl ether[J]. Journal of Applied Polymer Science,2020,137(34). 